\title{
Tratamiento de Flegmasía Cerúlea Dolens con Trombólisis; Reporte de caso.
}

Peña Peralta $L A^{1}$, Mora Posada $L R^{2}$, Hernández Forero $C G^{3}$, Villegas González $F^{4}$.

1. Médico y Cirujano General Fundación Universitaria Juan N. Corpas, Médica rotatoria en Cirugía Vascular del Hospital Departamental Universitario del Quindio San Juan de Dios Armenia y Departamento de Cirugía Vascular en Clínica La Sangrada Familia, Armenia - Colombia.

2. Médico Cirujano Vascular Periférico, Fundación Universitaria de Ciencias de la Salud Hospital de San José, Hospital Departamental Universitario del Quindio San Juan de Dios Armenia y Departamento de Cirugía Vascular en Clínica La Sangrada Familia, Miembro de la Asociación de cirugía vascular y angiología, Armenia-Colombia

3. Médico Cirujano Vascular y Angiología, Universidad Militar Nueva Granada, Terapia Endovascular, Hospital Clínico Valladolid - España, Flebología Clínica, Universidad del Bosque Bogotá - Colombia, Instructor de Cirugía con Radiofrecuencia en Colombia y Latinoamérica. Hospital Departamental Universitario del Quindio San Juan de Dios Armenia y Departamento de Cirugía Vascular en Clínica La Sangrada Familia, Miembro de la Asociación de cirugía vascular y angiología, Armenia - Colombia.

4. Médico Cirujano Cardiovascular Pontificia Universidad Católica Porto alegre - Brasil. Hospital Departamental Universitario del Quindio San Juan de Dios Armenia y Departamento de Cirugía Vascular en Clínica La Sangrada Familia, Miembro de la Asociación de cirugía vascular y angiología, Armenia - Colombia.

Autor de correspondencia: lauraa-pena@juanncorpas.edu.co

Como citar: Peña Peralta LA, Mora Posada LR, Hernández Forero CG, Villegas González F. Tratamiento de Flegmasía Cerúlea Dolens con Trombólisis; Reporte de caso. Revista Cuarzo 2018: 24 (2) 44- 49.

Recibido: 12 de agosto de 2018 Aceptado: 26 de octubre de 2018 Publicado: 30 de diciembre de 2018

\section{Resumen}

La Flegmasía Cerúlea Dolens es una complicación rara y severa de la trombosis venosa profunda, que se manifiesta clínicamente con edema profuso, dolor y cianosis del miembro inferior afectado. La obstrucción masiva del sistema venoso determina un aumento de la presión de los compartimentos de la extremidad, que finalmente compromete la circulación arterial. Su tratamiento debe ser agresivo para evitar la gangrena y/o la muerte. Presentamos el caso de una paciente atendida en la Clínica La Sagrada Familia, Armenia - Colombia, quien consultó por cuadro clínico de edema progresivo en miembro inferior izquierdo, encontrando cambios isquémicos en falanges distales y edema grado III en miembro inferior izquierdo, con reporte de dúplex venoso con trombosis venosa profunda iliofemoral extensa izquierda y dúplex arterial con reducción de los flujos arteriales secundario a edema severo de tejidos blandos, se indica flebografía con trombólisis venosa por catéter regional en miembro inferior izquierdo con infusión continua de trombolítico. Con respectivos controles angiográficos a las 24 y 48 horas con recanalización del 80\% de las venas iliaca, femoral y poplítea, se suspendió infusión y se retiró catéter, continuó anticoagulación con heparina no fraccionada hasta lograr paso a anticoagulación oral con posterior egreso hospitalario dado su evolución satisfactoria.

Palabras Clave: Trombosis, edema, isquemia, anticoagulantes, terapia trombolítica.

\section{Treatment of Phlegmasia Cerulea Dolens with Thrombolysis; Case report.}

\begin{abstract}
The Flegmasia Cerulea Dolens is a rare and severe complication of deep venous thrombosis, which manifests clinically with profuse edema, pain and cyanosis of the affected lower limb. The massive obstruction of the venous system is an increase in the pressure of the limb compartments, which compromises the arterial circulation. Your treatment should be aggressive to avoid gangrene and / or death. We present the case of a patient treated at La Sagrada Familia Clinic, Armenia - Colombia, who consulted for clinical picture of progressive edema in left lower limb, finding ischemic changes in distal phalanges and grade III edema in left lower limb, with report of duplex venous with deep left iliofemoral venous thrombosis and arterial duplex with reduction of arterial flow secondary to severe soft tissue edema, phlebography with venous thrombolysis is indicated by regional catheter in left lower limb with continuous thrombolytic infusion. With the respective angiographic controls at 24 and 48 hours with $80 \%$ recanalization of the iliac, femoral and popliteal veins, the infusion was interrupted and the catheter was withdrawn, anticoagulation with unfractionated heparin was continued until oral anticoagulation was administered, the hospital discharge was performed given its satisfactory evolution.
\end{abstract}

Keywords: Thrombosis, edema, ischemia, anticoagulants, thrombolytic therapy.
Licencia creative commons

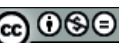




\section{Introducción}

Flegmasía cerúlea dolens (FCD) es una complicación poco habitual, fulminante y mortal de la trombosis venosa profunda (TVP) que lleva a una oclusión del flujo venoso de la extremidad inferior que puede conducir a compromiso arterial, isquemia tisular y gangrena.

Se atribuye a Fabricus Hildanus la identificación de la gangrena después de una trombosis venosa (1) en 1593, pero fue Cruveilhier (2) quien describiera que la relación fisiopatológica entre las trombosis grandes y pequeñas venas. En 1924, Buerger (3) reiteró la teoría de la oclusión venosa masiva y aclaró el cuadro clínico. Gregoire (4) en 1938 describió la tríada clínica consistente en hinchazón de los miembros, dolor isquémico agudo y decoloración, y acuñó el término "Flegmasía Cerúlea Dolens", que conduce a la congestión venosa con secuestro de líquidos masivo que conduce al colapso circulatorio y shock que causa la muerte.

Clásicamente se han descrito diferentes formas de presentación: la flegmasía alba dolens, donde hay una trombosis masiva del sistema venoso profundo, pero con las venas colaterales permeables y la flegmasía cerúlea dolens, en que la obstrucción también compromete a las venas colaterales. Dentro de la fisiopatología se dice que existe un estado de hipercoagulabilidad que genera la trombosis masiva precoz (las venas superficiales y las profundas), el edema, el aumento de la presión intersticial, un choque hipovolémico, la estenosis veno-capilar, la cianosis del miembro y la detención del flujo sanguíneo microvascular, que si es un bloqueo incompleto aparece la flegmasía cerúlea dolens (reversible) y si el bloqueo es completo con un cierre crítico, aparece la gangrena en el 40 a $60 \%$ de los casos (irreversible). (5) Cuadro clínico clásico está caracterizado por un dolor isquémico agudo, seguido de edema y cianosis del miembro inferior afectado.

Dentro de los factores desencadenantes del cuadro de trombosis masiva se encuentran los estados de hipercoagulabilidad como son: los síndromes paraneoplásicos, la trombocitopenia inducida por la heparina, el síndrome antifosfolipído, las cirugías recientes, las infecciones, la falla cardíaca y el embarazo (6), así como factores anatómicos o traumas predisponentes como el cateterismo venoso central, el síndrome de compresión ilio-cava.

El síndrome ilio-cavo, de Cockett o de May-Thurner es una condición anatómica en la cual la vena ilíaca común izquierda se encuentra comprimida a su paso entre la arteria ilíaca común derecha y la columna a la altura del cuerpo vertebral de la quinta vértebra lumbar o el promontorio del sacro justo antes de la unión ilio-cava con la consecuente aparición de trombosis venosa profunda en la extremidad inferior izquierda, lo cual puede llevar a insuficiencia venosa crónica o trombosis repetidas a largo plazo. La compresión iliocava se encuentra relacionada hasta con el $80 \%$ de las trombosis ilio-femorales (7).

La flegmasia se puede presentar a cualquier edad con casos reportados entre los seis meses y los 87 años, pero principalmen- te entre los 40 y 49 años; con mayor prevalencia en mujeres y siendo la extremidad izquierda la más afectada, en relación al síndrome de compresión iliocava. La tasa de mortalidad es tan alta como el $41 \%$ y la amputación definitiva puede ser necesaria en el 50\% de los supervivientes. (8) El diagnóstico es fundamentalmente clínico y requiere solo la confirmación mediante eco duplex.

Se han sugerido varias opciones de tratamiento para prevenir la morbimortalidad, tipificadas por el inicio de la anticoagulación sistémica, seguida por trombectomía quirúrgica con o sin fasciotomía, trombólisis dirigida por catéter, angioplastia transluminal percutánea con o sin colocación de stent, bypass venoso, trombectomía mecánica percutánea, dispositivos de aspiración o una combinación de estos. La FCD es una emergencia que requiere diagnóstico y tratamiento inmediato y agresivo para prevenir la gangrena venosa, una amputación o incluso la muerte. (9)

\section{Presentación Del Caso}

Paciente de sexo femenino de 86 años con antecedentes de Enfermedad Pulmonar Obstructiva Crónica oxígeno dependiente en tratamiento con Teofilina $100 \mathrm{mg}$ día y Sindenafil $50 \mathrm{mg}$ día, Insuficiencia Cardiaca Congestiva, Hipertensión Arterial y Arritmia en tratamiento con Losartan 50mg cada 12 horas, Amlodipino $5 \mathrm{mg}$ día, ácido acetilsalicílico $100 \mathrm{mg}$ día y Atorvastatina $40 \mathrm{mg}$ noche. Consulta al servicio de urgencias de la Clínica La Sagrada Familia de Armenia, por cuadro clínico de 3 días de evolución consistente en edema en miembro inferior izquierdo asociado a dolor y cianosis.

Al examen físico alerta y orientada, sin signos de dificultad respiratoria, cardiopulmonar con estertores ocasionales, extremidades resalta edema masivo, coloración violácea y dolor severo a nivel de los artejos, signos de isquemia en antepié dados por cambios a nivel de las falanges distales del 2 y 5 to artejos, con marcada disminución en los pulsos distales (Figura 1).

Se realiza dúplex venoso mostrando una trombosis venosa profunda masiva a nivel iliofemoral extensa izquierda. Se hospitaliza para manejo, realizándose flebografía que demuestra presencia de múltiples defectos de llenado a nivel de las venas poplítea, femoral, común, iliaca externa e iliaca común en relación a trombosis de aspecto agudo (Figura 2). Se propone entonces realizar trombolisis venosa regional del miembro inferior izquierdo que la paciente acepta.

Se realiza punción eco-guiada de vena poplítea izquierda, se ubica catéter de Fountaine en vena iliaca común dejando infusión continua de actilyse (alteplasa) a dosis de $0.01 \mathrm{mg} / \mathrm{kg} / 24 \mathrm{~h}$ vía catéter regional durante las siguientes 48 horas, adicionalmente infusión de heparina no fraccionada por el introductor en vena poplítea a una dosis de $300 \mathrm{U} / \mathrm{h}$, se traslada a UCI para realizar monitoreo. 

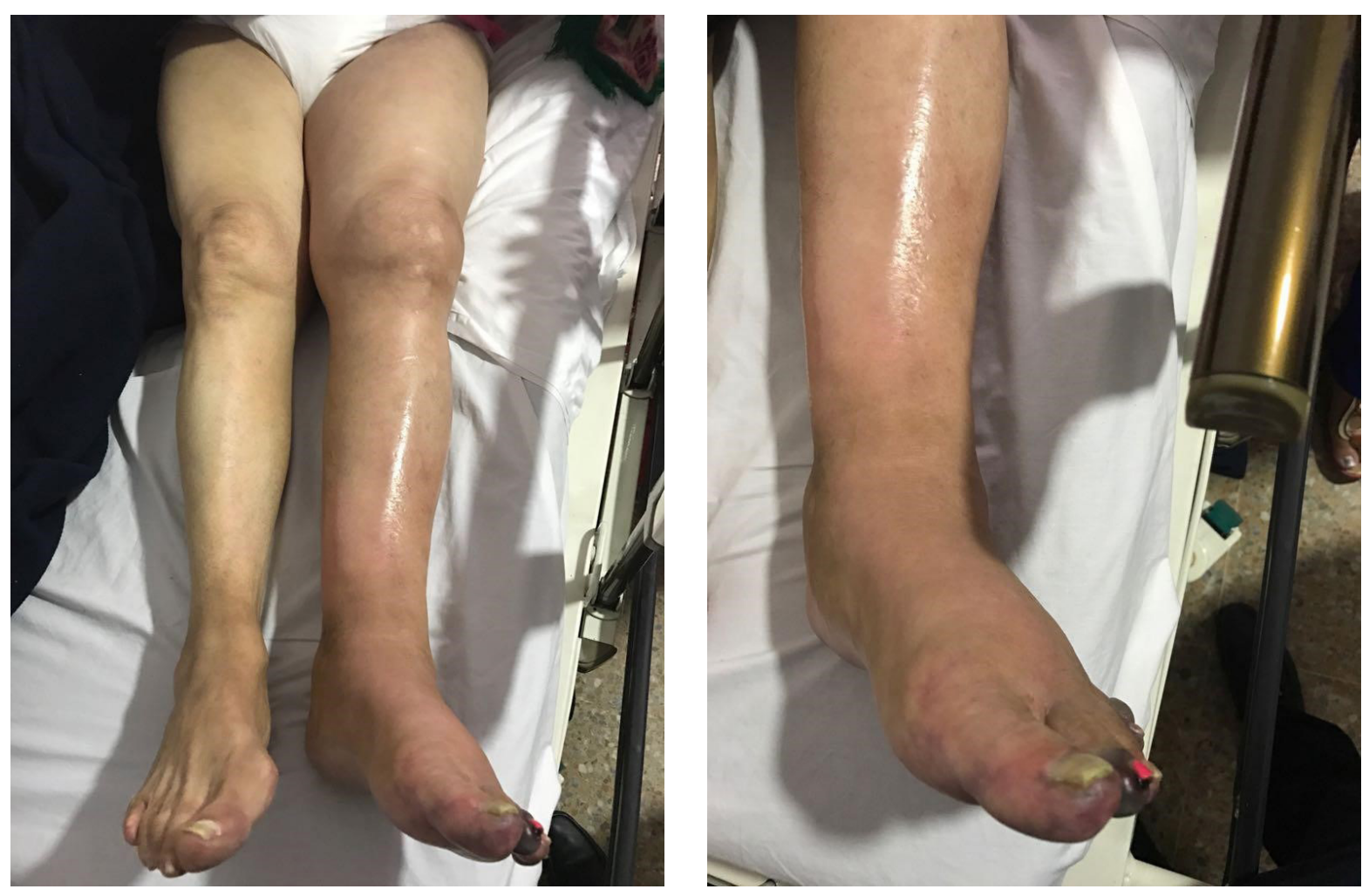

Figura I. Miembro inferior izquierdo presentación de la Flegmasía Cerúlea Dolens,

A las 24 horas se realiza control angiográfico con hallazgos de una recanalización del $60 \%$ de vena poplítea y femoral superficial izquierda; A nivel de las venas iliacas recanalización del $50 \%$ de venas iliaca común y externa izquierda. Al examen

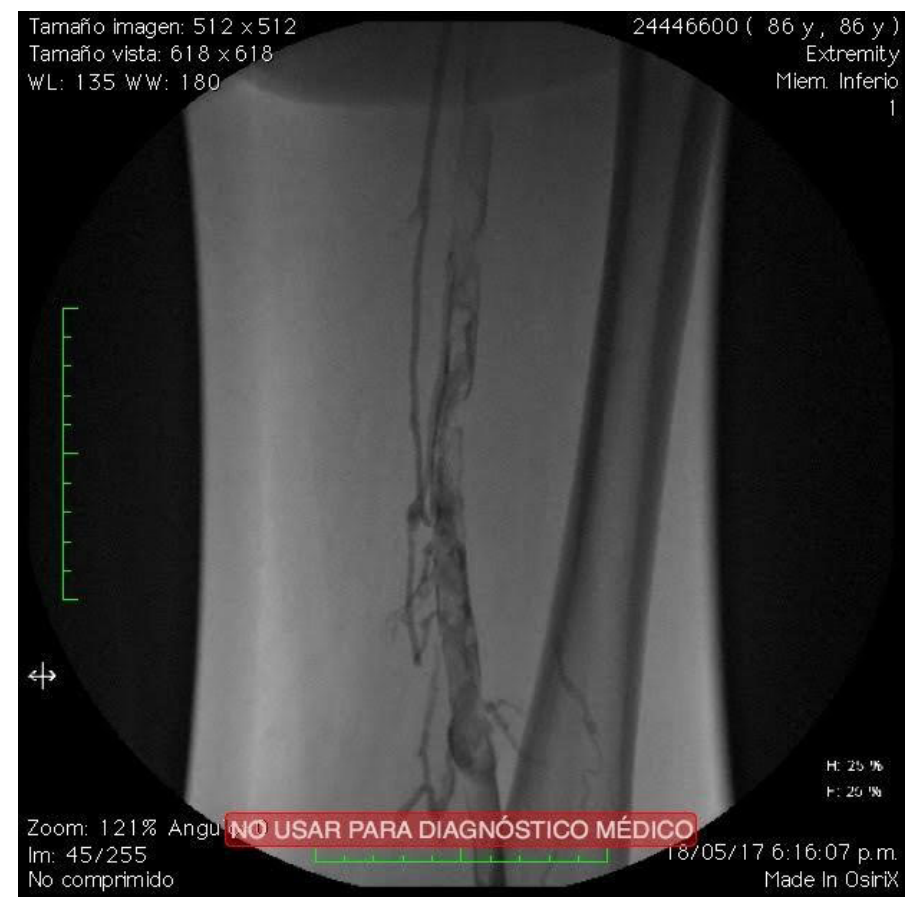

físico se observa la extremidad con un llenado capilar menor a 2 segundos, con marcada disminución del edema y el dolor. Se continúa igual infusión de actilyse y se espera nuevo control angiográfico.

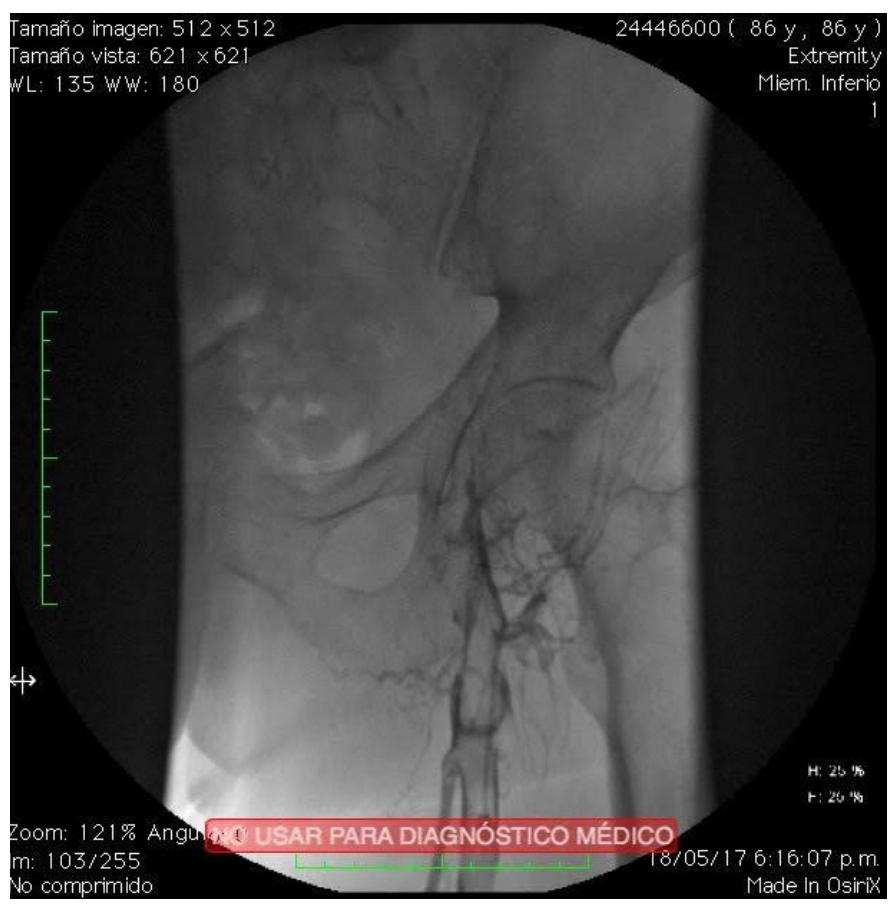

Figura 2. Flebografía e ileocavografía inicial. 
Al siguiente día se observa en la flebografía una recanalización del $80 \%$ de la vena poplítea y femoral superficial izquierda, y por último en la ileocavografía se evidencia una recanalización del $80 \%$ de vena iliaca común y externa (Figura 3 ), por lo anterior se retira catéter e introductor poplíteo, se suspende infusión de actilyse y se continúa heparina no fraccionada a $600 \mathrm{u} / \mathrm{h}$, manteniendo un tiempo parcial de tromboplastina en un rango de $1,5-2,5$ veces.
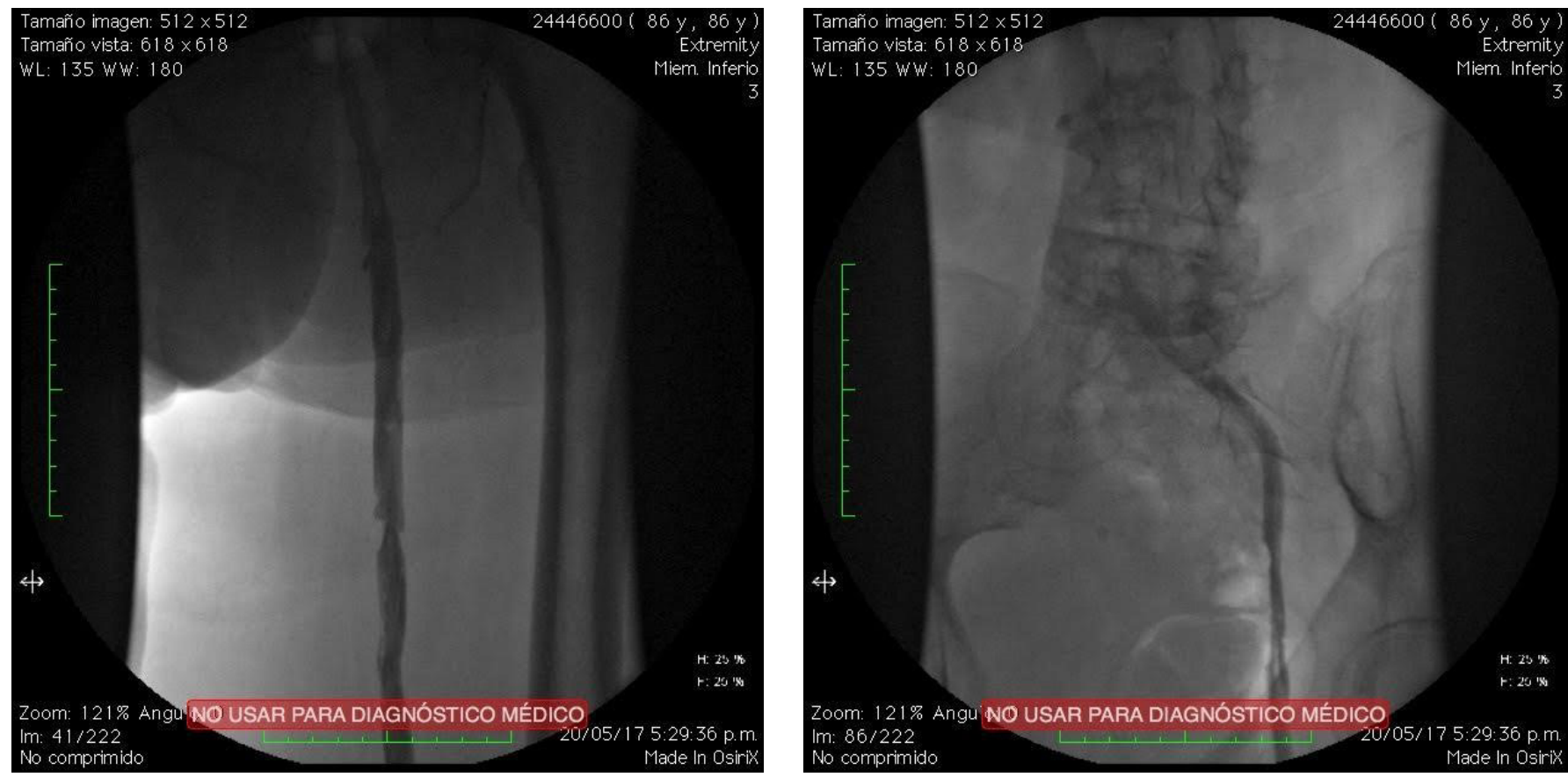

Figura 3. Flebografía e ileocavografía a las 48 horas.

La evolución fue satisfactoria y muy rápida dada por la mejoría de síntomas y ausencia de nuevos cambios isquémicos (Figura 4), por lo que al día $7 \mathrm{mo}$ de hospitalización se realizó el cambio a terapia anticoagulante oral (Dabigatran) y al día 8vo se da egreso hospitalario continuando anticoagulación oral y controles con cirugía vascular de forma ambulatoria.
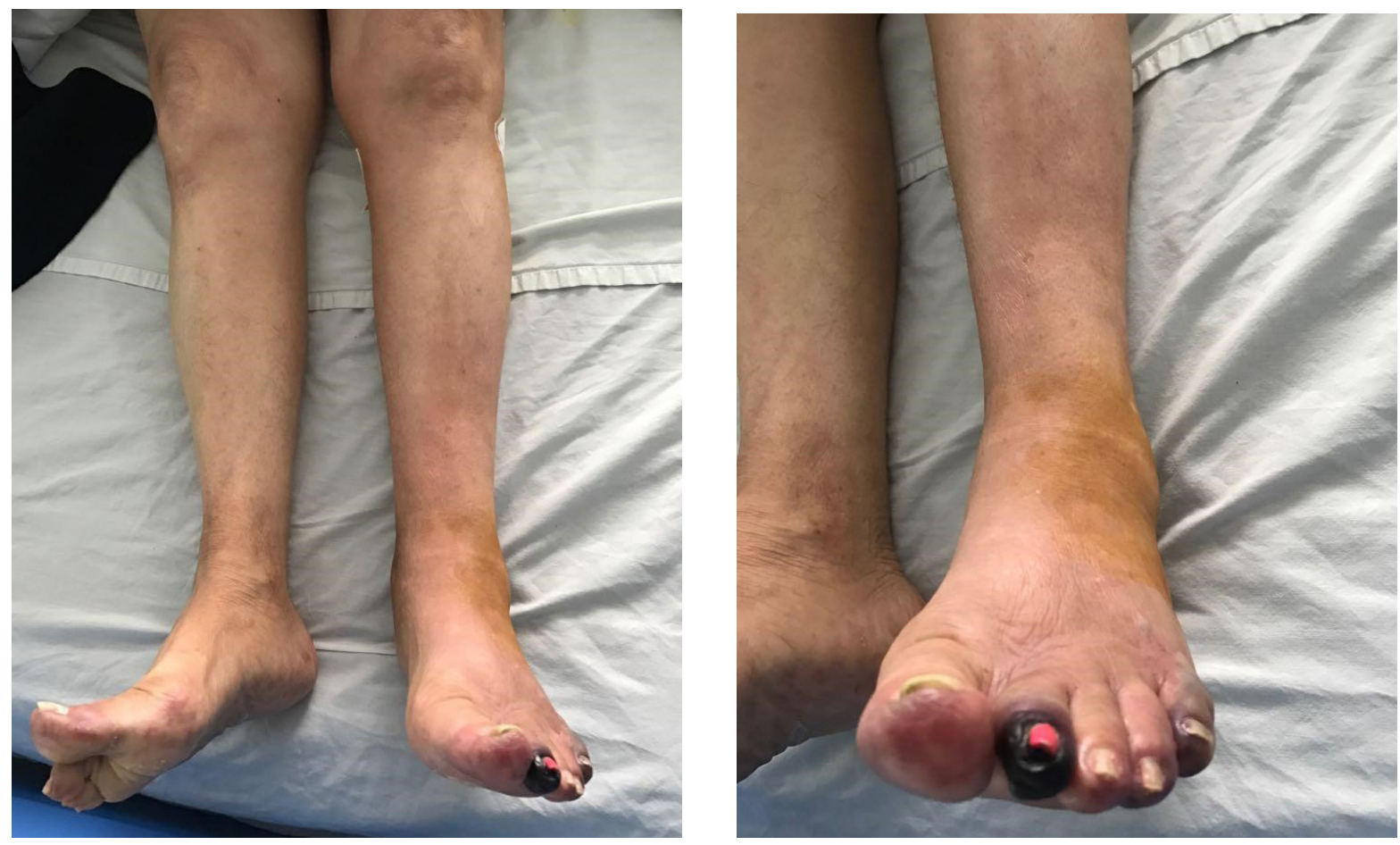

Figura 4. Miembro inferior izquierdo luego de trombólisis venosa. 


\section{Discusión}

La flegmasía Cerúlea Dolens es la complicación más severa a nivel local de la trombosis venosa profunda, siendo fuente de alta morbilidad y mortalidad. El objetivo del tratamiento es limitar la progresión del trombo, disminuir la hipertensión venosa y evitar la gangrena de la extremidad, además de restaurar el flujo sanguíneo y la protección de las válvulas venosas. Entre las alternativas terapéuticas para su manejo encontramos la anticoagulación con elevación de la extremidad y reposición de volumen, la trombólisis, la trombo-aspiración y la tromboembolectomía quirúrgica, en los casos en que se reporta síndrome compartimental se debe incluir la fasciotomía.

En el tratamiento médico se habla de Anticoagulación sistémica donde se recomienda usar heparina no fraccionada por la velocidad con la que se logra la anticoagulación del paciente, es crucial en el manejo, pero se considera un tratamiento insuficiente en la mayoría de los casos dada la severidad del cuadro.

Con respecto al tratamiento quirúrgico, la tromboembolectomía venosa según la literatura muestra que en sus primeros estudios los resultados eran desfavorables, puesto que es una técnica más difícil que la tromboembolectomía arterial con mayor pérdidas de sangre y menos posibilidades de eliminación completa de los trombos, por la friabilidad del mismo y la presencia de válvulas que impiden u obstruyen el paso del catéter, además de correr un riesgo significativo de embolismo pulmonar y tasas de mortalidad del $9 \%$. (10)

Sobre las técnicas endovasculares, las técnicas preferidas usualmente son la trombólisis y la trombo-aspiración. El uso de trombolíticos para el tratamiento de FCD se describió por primera vez en 1970. El primer trombolítico estudiado fue estreptoquinasa, y la primera serie publicada mostró excelentes resultados (11). Hoy en día se dice que no se utilizan de forma sistémica por su escasa respuesta y reportes de mayores complicaciones, La trombólisis dirigida por catéter (TDC) se refiere a la infusión de un agente trombolítico directamente en el trombo venoso a través de un catéter de múltiples orificios. Previamente al procedimiento se revisan las imágenes para determinar el sitio de acceso apropiado, con el fin de maximizar la infusión de agentes trombolíticos en toda la extensión del trombo. Las opciones de acceso incluyen las venas femorales común, yugular interna, poplítea o tibial posterior.

Existen diferentes trombolíticos (uroquinasa y rt-PA, uno de los más usados) la elección entre uno y otro, se han hecho sobre la base de la experiencia local, la eficacia y el perfil de seguridad con respecto al riesgo de complicaciones hemorrágicas graves, pero no hay estudios que demuestren la diferencia en esta patología. En un estudio encontraron que TDC más anticoagulación daba como resultado una mejor permeabilidad venosa a los 6 meses, menos obstrucción venosa funcional y ninguna diferencia en el reflujo venoso femoropoplíteo en comparación con el anticoagulante solo (12), con respecto al caso presentado en don- de se usó TDC y anticoagulación los resultados clínicos fueron en menos tiempo y sin complicaciones.

En informes de estudios anteriores revelaron resultados exitosos de trombólisis dirigida por catéter en el tratamiento de FCD, dados por la restauración de los pulsos arteriales y salvamento de la extremidad, en un $60 \%$ en pacientes con gangrena venosa y un $100 \%$ sin gangrena venosa (13). Adicionalmente existen complicaciones con respecto al procedimiento tales como; alteraciones neurológicas, hemorragias, hematomas, fiebre, náuseas, vómitos y muerte.

Una revisión sistemática realizada por Casey et al. Concluyó que al comparar la trombólisis sistémica con trombólisis dirigida por catéter se asoció con una reducción significativa en el riesgo de síndrome post-trombótico y obstrucción venosa (14). Siendo así una forma alternativa efectiva para la lisis del trombo con bajas complicaciones.

La trombectomía por aspiración manual hace referencia a la eliminación mecánica del trombo mediante la aplicación de succión a través de un catéter u otro dispositivo, es la forma más simple en la que utilizan la presión negativa a través de una jeringa unida al catéter. Estudios previos han reportado índices de éxito del 96-100\% (15).

La trombectomía asistida por vacío es similar a la trombectomía por aspiración manual. La diferencia es que la succión se aplica al catéter a través de una bomba de vacío externa conectada a este, utilizando un circuito veno-venoso extracorpóreo que coloca un filtro en serie con una cánula de aspiración y una cánula de reinfusión. Esta técnica permite que la trombectomía por aspiración prolongada continúe sin el riesgo de pérdida de sangre. Aunque no hay datos disponibles que demuestren la seguridad y la eficacia del dispositivo en el tratamiento de la trombosis venosa profunda aguda.

Ya para concluir La Flegmasia Cerulea Dolens es una entidad rara, pero de gran severidad. Su diagnóstico es fundamentalmente clínico y el tratamiento debe ser temprano y agresivo mediante anticoagulación sistémica y eliminación de la oclusión venosa, preferiblemente mediante técnicas endovasculares, es fundamental para evitar la gangrena, con pérdida de la extremidad y muerte, todo con el fin de evitar afectaciones en calidad de vida de los pacientes.

\section{Bibliografía}

1. HAIMOVICI, H. (1950). Gangrene of the Extremities of Venous Origin: Review of the Literature with Case Reports. Circulation, 1(2), pp.225-240.

2. Scirp.org. (2018). Buerger, L. (1926) The Circulatory Disturbances of the Extremities. Annals of Surgery, 83, 157. - References - Scientific Research Publishing. [online] Available at: http://www.scirp.org/reference/ReferencesPapers.aspx?ReferenceID=1404953 [Accessed 15 May 2018].

3. Emedicine.medscape.com. (2018). Phlegmasia Alba and Cerulea Dolens Clinical Presentation: History and Physical Examination. [online] Availa- 
ble at: https://emedicine.medscape.com/article/461809-clinical [Accessed 15 May 2018].

4. Chinsakchai, K., ten Duis, K., Moll, F. and de Borst, G. (2010). Trends in Management of Phlegmasia Cerulea Dolens. Vascular and Endovascular Surgery, 45(1), pp.5-14.

5. Ascaño Ortega, A. (2018). Flegmasía cerulea dolens. [online] Scielo.sld. cu. Available at: http://scielo.sld.cu/scielo.php?script=sci_abstract\&pi$\mathrm{d}=\mathrm{S} 1682-00372014000100009 \& \operatorname{lng}=\mathrm{en}$ [Accessed 15 May 2018].

6. Morán, N., Zegpi, B. (2011). Flegmasía Cerúlea Dolens: Diagnóstico y tratamiento. REV ANACEM, (VOL. $5 \mathrm{~N}^{\circ}$ 2), pp.109-111.

7. Butros, S., Liu, R., Oliveira, G., Ganguli, S. and Kalva, S. (2013). Venous compression syndromes: clinical features, imaging findings and management. The British Journal of Radiology, 86(1030), p.20130284.

8. Kuo, I., Smith, J. and Abou-Zamzam, A. (2011). A multimodal therapeutic approach to phlegmasia cerulea dolens in a pediatric patient. Journal of Vascular Surgery, 53(1), pp.212-215.

9. Oguzkurt, L., Ozkan, U., Demirturk, O. and Gur, S. (2010). Endovascular Treatment of Phlegmasia Cerulea Dolens with Impending Venous Gangrene: Manual Aspiration Thrombectomy as the First-Line Thrombus Removal Method. CardioVascular and Interventional Radiology, 34(6), pp.12141221.

10. Rutherford R., Eklof B., Mewissen M. P (2000). Interventional treatments for iliofemoral venous thrombosis. Rutherford's Vascular Surgery. 5th ed. W.B.Saunders; p. 1959-68.

11. Mousa, A., Henderson, P., Dayal, R., Bernheim, J., Kent, K. and Faries, P. (2005). Endoluminal Recanalization in a Patient with Phlegmasia Cerulea Dolens Using a Multimodality Approach. Vascular, 13(05), p.313.

12. Weaver, F., Meacham, P., Adkins, R. and Dean, R. (1988). Phlegmasia Cerulea Dolens: Therapeutic Considerations. Southern Medical Journal, 81(3), pp.306-312.

13. Patel N., Plorde J., Meissner M. (1998) Trombólisis dirigida por catéter en el tratamiento de flegmasía cerúlea dolorosa. Ann Vasc Surg; 12 (5): 471-5

14. Casey E., Murad M., García M., Elamin M, (2012) El tratamiento de la trombosis venosa profunda iliofemoral aguda. J Vasc Surg; 55 (5): 1463-73

15. Oguzkurt, L., Ozkan, U., Gulcan, O., Koca, N. and Gur, S. (2011). Endovascular treatment of acute and subacute iliofemoral deep venous thrombosis using manual aspiration thrombectomy: long-term result of 139 patients from a single center. Diagnostic and Interventional Radiology 18: 410-6. 